\title{
Review
}

Cerebrovascular Diseases
Cerebrovasc Dis 2013;35:23-39

DOI: $\underline{10.1159 / 000346076}$
Received: July 24, 2012

Accepted: November 22, 2012

Published online: February 14, 2013

\section{Apathy Secondary to Stroke: A Systematic Review and Meta-Analysis}

\author{
Lara Caeiro $^{a}$ José M. Ferro ${ }^{b}$ João Costa ${ }^{c}$ \\ ${ }^{a}$ Faculty of Medicine, Institute of Molecular Medicine, ${ }^{b}$ Stroke Unit, Neurology Service, Department of \\ Neurosciences, Hospital de Santa Maria and ' Laboratory of Clinical Pharmacology and Therapeutics, Center for \\ Evidence-Based Medicine and Cochrane Coordinating Center Portugal, Faculty of Medicine, University of Lisbon, \\ Lisbon, Portugal
}

\section{Key Words}

Systematic review $\cdot$ Meta-analysis $\cdot$ Apathy $\cdot$ Cognitive impairment $\cdot$ Depression $\cdot$ Stroke

\begin{abstract}
Background: Apathy is a disturbance of motivation, frequent in survivors of stroke. Several studies have evaluated the rate of apathy secondary to stroke and risk factors. Different conclusions and contradictory findings have been published. We aimed to perform a systematic review and meta-analysis of all studies evaluating apathy secondary to stroke to better estimate its rate and risk factors, and explore associations with poorer outcomes. Methods: We searched PubMed, Cochrane Library, PsychINFO and PsycBITE databases and screened references of included studies and review articles for additional citations. Search results and data extraction was performed independently. We systematically reviewed available publications reporting investigations on ischemic and intracerebral hemorrhagic stroke and apathy. Quality assessment of the studies was performed independently. Subgroup analyses were performed according to stroke phase (acute and post-acute), stroke past history (firstever and any-stroke) and patient age (younger and older patients). Pooled odds ratios (OR) and standardized mean dif-
\end{abstract}

ference, and $95 \%$ confidence intervals $(\mathrm{Cl})$, were derived by random-effects meta-analysis. Heterogeneity was assessed with $1^{2}$ test. Results: From the initial 1,399 publications, we included 19 studies (2,221 patients). The pooled rate of apathy was $36.3 \%\left(95 \% \mathrm{Cl} 30.3-42.8 ; 1^{2}=46.8\right)$, which was similar for acute $[39.5 \%(95 \% \mathrm{Cl} 28.9-51.1)]$ and post-acute phase [34.3\% (95\% Cl 27.8-41.4)], and about three times higher than the rate of depression [12.1\% $(95 \% \mathrm{Cl} 8.2-17.3)]$. Apathetic patients were on average 2.74 years older $(95 \% \mathrm{Cl}$ $\left.1.25-4.23 ; I^{2}=0 \%\right)$. No gender differences were found. Depression (OR $2.29 ; 95 \% \mathrm{Cl} 1.41-3.72 ; \mathrm{I}^{2}=44 \%$ ) and cognitive impairment (OR 2.90; 95\% Cl 1.09-7.72; $\left.\mathrm{I}^{2}=14 \%\right)$ were more frequent and severe in apathetic patients. Apathy rate was similar for ischemic and hemorrhagic stroke type and for left- and right-sided hemispheric lesions. Clinical global outcome was similar between apathetic and nonapathetic patients. Conclusion: Apathy secondary to stroke is a more frequent neuropsychiatric disturbance than depression. Apathetic patients are more frequently and severely depressed and cognitively impaired. A negative impact of apathy secondary to stroke on clinical global outcome cannot be ascribed. Future research should properly address its predictor factors and evaluate the impact of apathy treatment options in stroke patients.

Copyright $\odot 2013$ S. Karger AG, Basel

\section{KARGER}

Fax +4161306 1234

E-Mail karger@karger.com

www.karger.com
(C) 2013 S. Karger AG, Basel

$1015-9770 / 13 / 0351-0023 \$ 38.00 / 0$

Accessible online at:

www.karger.com/ced
Lara Caeiro

Stroke Unit, Serviço de Neurologia, Hospital de Santa Maria

Avenida Professor Egas Moniz

PT-1649-035 Lisbon (Portugal)

E-Maillaracaeiro@fm.ul.pt 


\section{Introduction}

Apathy is a disturbance of motivation evidenced by diminished goal-directed overt behavior, diminished goal-directed cognition and diminished emotional concomitants of goal-directed behavior [1,2]. Apathy is thought to be a frequent complication of stroke and a disabling and stressor condition, both for patients and caregivers [3, 4]. Recently, several studies have been conducted to specifically evaluate the rate of apathy secondary to stroke, either in the acute or post-acute phases. However, these studies reached different conclusions and contradictory findings have been published [5-7]. Therefore, we aimed to perform a systematic review and meta-analysis of all studies that evaluated apathy secondary to stroke to better estimate its rate and relationship with associated factors, as well as to explore whether apathy is associated with a poorer clinical outcome.

\section{Methods}

For the purposes of this systematic review we took the MOOSE statement as a guideline [8].

\section{Eligibility Criteria}

Our primary objective was the rate of apathy among stroke patients. Secondary objectives were to explore associations between apathy and age, gender, stroke location (hemispheric left/ right) and type (ischemic or intracerebral hemorrhage), depression, cognitive impairment and clinical outcome.

Cases of apathy were all considered for analysis provided that apathy was assessed with validated scales or through clearly defined criteria. Studies that did not specifically mention which validated scales were used for assessing apathy were included, but we performed sensitivity analysis to evaluate the impact of their results in the outcome results. If studies reported only data for cases described as apathy-related disturbances (e.g. abulia, akinesia, mutism, avolition, athymhormia, self-activation, indifference), these were only considered for analysis providing that the definition of these cases clearly referred to diminished motivation, lack of emotion, interest or concern $[2,9,10]$.

We considered all types of observational studies or case series. However, the potential risk of bias from selective reporting led us to include only studies in stroke patients that specifically evaluated apathy, either as their primary objective or as a prespecified outcome. Only ischemic or intracerebral hemorrhagic stroke patients were considered, irrespective of risk factors. Studies enrolling patients with nonvascular cerebral diseases or any type of dementia were excluded. We also excluded case series with less than 10 patients and those including only patients with specific focal stroke locations. This minimum number was chosen to exclude single case reports and small series, therefore decreasing the risk of selection bias.

To decrease detection bias we only included studies based on institutions, either rehabilitation or hospital populations. We ex- cluded community-based studies reporting apathy secondary to silent or asymptomatic stroke. We also excluded the following publications: (1) focusing on the metric properties of scales to assess apathy, with no report on apathy rate secondary to stroke; (2) investigating the relationship between apathy secondary to stroke and silent strokes, white matter lesions or other vascular neuroimaging findings, and (3) with stroke patients submitted to brain surgery or endovascular treatment.

\section{Information Sources and Search Method}

Potentially eligible studies were identified through an electronic search of bibliographic databases from inception to April 2012 (Medline through PubMed, Cochrane Library databases, PsychINFO and PsycBITE) and by extensive searching using cross-references from original articles and reviews.

The search used the following terms: stroke OR [(ischemia OR infarction OR accident) and (cerebr* OR brain OR hemisphere OR subcortical OR cortical)] OR cerebrovascular disorders combined with apath* and apathy-related key-words (abulia OR akinesia OR mutism OR avolition OR athymhormia OR self-activation OR indifference). All terms were searched as indexed and as free text terms to increase sensitivity. The search was limited to studies conducted on adult humans and published in English, German, French, Spanish or Portuguese. We screened titles, keywords and abstracts of the citations downloaded from the electronic searches and obtained full copies of potentially suitable reports for further assessment. For publications with unclear or incomplete data, we contacted the authors asking for further information.

\section{Study Selection and Data Collection Process}

Titles and abstracts of obtained records were screened. Doubts and disagreements were solved by consensus. Selected studies were assessed in full-text to determine their appropriateness for inclusion. Two authors independently extracted data from study design, location, time-frame of study, patient characteristics, study primary outcome and data of required outcomes.

When two or more publications referring to the same sample were available, we extracted data only from the publication presenting the most accurate estimate, either because of sample size or outcome assessment. For our analysis we used the risk estimate reported in each publication for apathy and associated factors. If no estimate was available, we derived the crude odds ratio (OR) from raw data.

Quality assessment of uncontrolled studies is an unsolved issue and debate exists about which should be used in the assessment of risk of bias in case-series studies [11]. We used a set of criteria derived from those suggested by the Centre for Reviews and Dissemination $[12,13]$ (online suppl. appendix 1; for all online suppl. material, see www.karger.com/doi/10.1159/000346076). The quality of reporting was independently analyzed by two authors.

\section{Statistics and Meta-Analysis}

We used Meta-Analyst [14] and Revman 5.1 [15] software for statistical analysis and to derive forest plots showing the results of individual studies and pooled analysis. Random-effects metaanalysis weighted by the inverse-variance method was performed to estimate pooled OR and 95\% confidence intervals (CI) [16]. Heterogeneity was assessed with the $\mathrm{I}^{2}$ test that measures the per- 
Fig. 1. Literature search and results (sys-

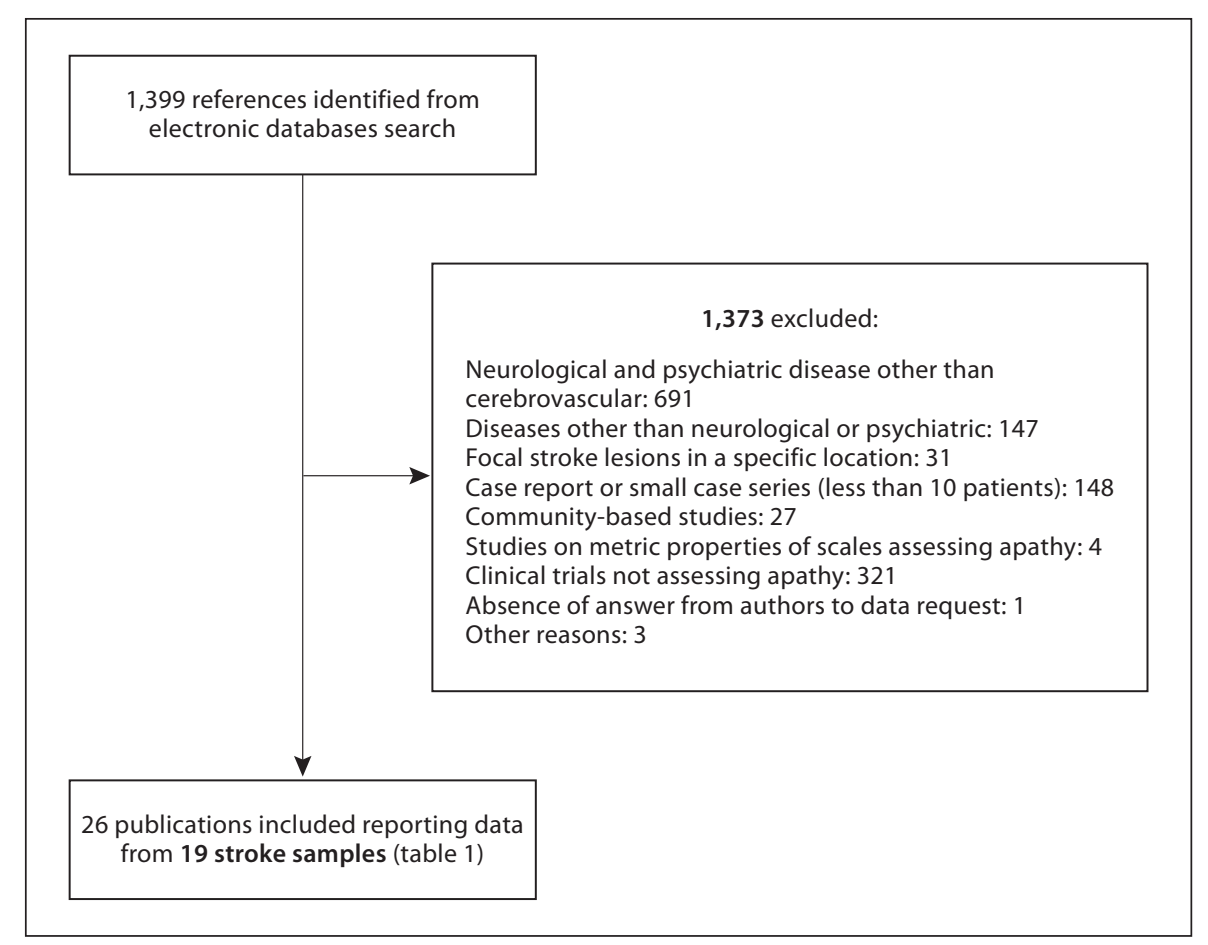
tematic review flow chart).

centage of total variation between studies due to heterogeneity [17]. We used a random-effects model independently of the existence $\left(\mathrm{I}^{2} \geq 50 \%\right)$ or not of substantial heterogeneity between study results. The effect measurement estimate chosen was OR because relative estimates are more similar across studies with different designs, populations and lengths of follow-up than absolute effects [18]. Raw data was first converted to OR through classic methods or through the Peto method if one arm had a zero-count cell. When raw data or OR were not available we took the hazard ratio or risk ratio for analysis. When significant associations were found, we determined the differences between apathetic and nonapathetic patients in corresponding rating scores using either the mean difference or the standardized mean difference (SMD) if studies used the same or different scales, respectively, to assess the outcome.

For primary outcome (apathy rate), we presented the results stratified according to stroke phase (acute and post-acute), stroke past history (first-ever and any-stroke) and patient age (younger and older patients) to explore differences in outcome estimates according to these patient subgroups. The cut-off time period used to classify a study as acute or post-acute phase was 15 days from stroke onset. The cut-off used for classifying patients as younger or older was 65 years (mean age). Differences between subgroups were tested using random effects meta-regression. When moderate-to-high heterogeneity $\left(\mathrm{I}^{2}>50 \%\right)$ existed in pooled estimates for the associations between apathy and related factors, we explored whether stroke phase, stroke past history or patient age could, at least partly, explain the heterogeneity. A sensitivity analysis excluding studies of poorer quality was conducted to explore the impact of the studies' quality on the results.

Apathy Secondary to Stroke: A Systematic Review

\section{Results}

\section{Search Results}

The electronic database search yielded a total of 1,399 publications. Following our inclusion and exclusion criteria, we were able to include 26 publications reporting data on apathy secondary to stroke. No study reporting data for cases described as apathy-related disturbances met our inclusion criteria. Twelve publications $[3,5,19-30]$ reported results for 6 stroke data samples. In these cases, to avoid double counting, we extracted data from the publication presenting the most accurate estimate for the outcome of interest. Therefore, a total of 19 different stroke samples (26 publications) were included for analysis. We excluded one study [31] because no data on the rate of apathy was provided in the publication and the authors did not provide further information after having been contacted. Figure 1 shows the detailed results of the search strategy.

\section{Description of Studies}

Not all studies that evaluated the rate of apathy were prospective. With the exception of two publications, data for our primary outcome could accurately be extracted directly from the publications. In these two cases with unclear data $[6,22,23]$, we contacted the authors and further clarification was provided. 
Table 1. Study characteristics

\begin{tabular}{|c|c|c|c|c|c|}
\hline Study & $\begin{array}{l}\mathrm{n}(\% \text { male }) \\
\text { Mean age }\end{array}$ & $\begin{array}{l}\text { Stroke type and lesion } \\
\text { location }\end{array}$ & Apathy & Apathy assessment & Study objectives \\
\hline \multicolumn{6}{|c|}{ Acute stroke ( $\leq 15$ days): hospital setting } \\
\hline Caeiro et al. $[5,30]$ & $\begin{array}{l}\text { Case-control } \\
94(64.9) \\
55.7[27-84]\end{array}$ & $\begin{array}{l}72(76.6) \text { infarcts } \\
22(23.4) \text { hemorrhages } \\
31(33) \text { left; } 37(39.4) \text { right } \\
69(73.4) \text { hemispheric } \\
25(26.6) \text { brainstem } \\
20(21.3) \text { cortical } \\
26(27.9) \text { subcortical }\end{array}$ & $36(38.3)$ & $\begin{array}{l}\text { Apathy evaluation scale - } \\
10 \text {-item clinical rate }\end{array}$ & $\begin{array}{l}\text { Associations between apathy } \\
\text { and stroke, cognitive } \\
\text { impairment, depression and } \\
\text { functional outcome }\end{array}$ \\
\hline $\begin{array}{l}\text { Carota et al. } \\
{[27-29]}\end{array}$ & $\begin{array}{l}\text { First-ever } \\
273(53) \\
64.4[19-90]\end{array}$ & $\begin{array}{l}273(100) \text { infarcts } \\
122 \text { (53) left; } 107(47) \text { right } \\
50(18.4) \text { infratentorial } \\
223(81.7) \text { supratentorial } \\
58(21.2) \text { subcortical } \\
165(60.4) \text { cortical }\end{array}$ & $130(48)$ & $\begin{array}{l}\text { Emotion behavior } \\
\text { Index form }\end{array}$ & $\begin{array}{l}\text { Associations between acute and } \\
\text { post-stroke depression (27) }\end{array}$ \\
\hline $\begin{array}{l}\text { Jarzebska } \\
2007[7]\end{array}$ & $\begin{array}{l}90(46.6) \\
53[28-72]\end{array}$ & 90 (100) infarcts & $64(71)$ & Apathy scale & $\begin{array}{l}\text { Frequency of apathy in acute } \\
\text { stroke, and the relationship with } \\
\text { stroke location and depression }\end{array}$ \\
\hline $\begin{array}{l}\text { Kang and Kim } \\
2008 \text { [39] }\end{array}$ & $\begin{array}{l}100(58) \\
65.1[29-88]\end{array}$ & $\begin{array}{l}100 \text { (100) infarcts } \\
55 \text { (55) left; } 45 \text { (45) right }\end{array}$ & $43(53)$ & Clinical observation & $\begin{array}{l}\text { Etiology, clinical and imaging } \\
\text { findings in ACA infarction }\end{array}$ \\
\hline $\begin{array}{l}\text { Onoda et al. } \\
2011[6]\end{array}$ & $\begin{array}{l}102(55.9) \\
73.2[41-96]\end{array}$ & $\begin{array}{l}102 \text { (100) infarcts } \\
45 \text { (44.1) left, } 41(40.2) \text { right } \\
13(12.7) \text { bilateral } \\
42(41.2) \text { cortical } \\
76(74.5) \text { subcortical }\end{array}$ & $37(36)$ & Apathy scale & $\begin{array}{l}\text { Relationship between post- } \\
\text { stroke apathy and regional } \\
\text { cerebral blood flow }\end{array}$ \\
\hline $\begin{array}{l}\text { Piamarta et al. } \\
2004[34]\end{array}$ & $\begin{array}{l}\text { First-ever } \\
33(60.6) \\
71.6[60-88]\end{array}$ & $\begin{array}{l}24(72.7) \text { infarcts } \\
9(27.3) \text { hemorrhages } \\
19(57.6) \text { left; } 14(42.4) \text { right } \\
5(15.2) \text { frontal } \\
16(48.5) \text { thalamus/basal } \\
\text { ganglia }\end{array}$ & $5(15.2)$ & $\begin{array}{l}\text { Clinical interview-based } \\
\text { questions }\end{array}$ & $\begin{array}{l}\text { Assess the presence of apathy, } \\
\text { anhedonia and emotional } \\
\text { lability and depression in first } \\
\text { ever supratentorial stroke }\end{array}$ \\
\hline $\begin{array}{l}\text { Starkstein et al. } \\
1993[9]\end{array}$ & $\begin{array}{l}\text { First-ever } \\
80(48.8) \\
60[-]\end{array}$ & Infarcts and hemorrhages & $18(22.5)$ & Apathy Scale & $\begin{array}{l}\text { Frequency and correlates of } \\
\text { apathy in stroke }\end{array}$ \\
\hline \multicolumn{6}{|c|}{ Post-acute stage (>15 days): hospital setting } \\
\hline $\begin{array}{l}\text { Angelelli et al. } \\
2004 \text { [37] }\end{array}$ & $\begin{array}{l}\text { First-ever } \\
124(71) \\
60.6[-]\end{array}$ & $\begin{array}{l}124 \text { (100) infarcts } \\
53 \text { (42.7) left; } 71 \text { (57.3) right }\end{array}$ & $33(26.6)$ & $\begin{array}{l}\text { Neuropsychiatric } \\
\text { Inventory-apathy }\end{array}$ & $\begin{array}{l}\text { Neuropsychiatric } \\
\text { symptomatology and its } \\
\text { evolution in stroke }\end{array}$ \\
\hline $\begin{array}{l}\text { Brodaty et al. } \\
{[25,26]}\end{array}$ & $\begin{array}{l}\text { Case-control } \\
135(60.7) \\
72.2[<85]\end{array}$ & 135 (100) infarcts & $36(26.7)$ & $\begin{array}{l}\text { Apathy evaluation } \\
\text { Scale informant }\end{array}$ & $\begin{array}{l}\text { Frequency and clinical correlates } \\
\text { of apathy in stroke }\end{array}$ \\
\hline $\begin{array}{l}\text { Glodzik-Sobanska } \\
2005[38]\end{array}$ & $\begin{array}{l}\text { Case-control } \\
\text { First-ever } \\
31(51.6) \\
62.9[45-80]\end{array}$ & $\begin{array}{l}31 \text { (100) infarcts } \\
16 \text { (51.6) left; } 15(48.4) \text { right } \\
31(100) \text { hemispheric } \\
4(12.9) \text { cortical } \\
18 \text { (50.1) subcortical } \\
9 \text { (29) cortico-subcortical }\end{array}$ & $13(42)$ & Apathy scale & $\begin{array}{l}\text { Biochemical changes in frontal } \\
\text { lobes and its correlation with } \\
\text { apathy }\end{array}$ \\
\hline $\begin{array}{l}\text { Kaji et al. } \\
2006[36]\end{array}$ & $\begin{array}{l}92(61) \\
64.6[32-85]\end{array}$ & Infarcts and hemorrhages & $37(40.2)$ & Apathy scale & $\begin{array}{l}\text { Prevalence and clinical } \\
\text { correlates of post-stroke } \\
\text { depression including apathy }\end{array}$ \\
\hline
\end{tabular}


TIA, other chronic diseases, deliriums, consciousness disturbances, dementia, aphasia, nonstroke

$\begin{array}{llllll}\text { Intracerebral } & \text { Hemisphere } & \text { No } & \text { No } & \text { No } & \text { No }\end{array}$

Right-sided
Yes $\leq 1$ day hospitalization, delirium, epilepsy, infectious, Parkinson, bilateral or multiple lesions, hemorrhage, severe leukoaraiosis, alcohol abuse, previous nonautonomy
Intracerebral

No hemorrhage [28]

$\begin{array}{lllllll}- & \text { NS } & \text { Internal carotid NS } & \text { NS } & \text { No } & \text { Yes } & \text { NS }\end{array}$
artery territory

\begin{tabular}{|c|c|c|c|c|c|c|c|}
\hline $\begin{array}{l}\text { Non-ACA stroke location, } \mathrm{SAH} \text {, } \\
\text { subcortical stroke }\end{array}$ & NS & $\begin{array}{l}\text { Frontal } \\
\text { Left-sided } \\
\text { Bilateral }\end{array}$ & NS & NS & NS & NS & NS \\
\hline $\begin{array}{l}\text { No lesion (mean difference), hemorrhage, } \\
\text { dementia, conscious disturbance, aphasia, } \\
\text { previous psychiatric illness }\end{array}$ & NS & $\begin{array}{l}\text { Left-sided } \\
\text { basal ganglia }\end{array}$ & No & No & Yes & Yes & No \\
\hline Previous stroke, nonverbal aphasia & No & No & Yes & NS & Yes & Yes & Yes \\
\hline $\begin{array}{l}>85 \text { years old, hemorrhage, TIA, delirium, } \\
\text { nonfluent in English, dementia, alcohol or } \\
\text { drug abuse, mental retardation, aphasia }\end{array}$ & NS & $\begin{array}{l}\text { Right-sided } \\
\text { Fronto- } \\
\text { subcortical } \\
\text { circuit }\end{array}$ & Yes & No & No & Yes & Yes \\
\hline $\begin{array}{l}\text { Hemorrhage, multiple stroke lesions, } \\
\text { frontal lobe lesion, }>80 \text { years old, aphasia, } \\
\text { poor clinical status, consciousness } \\
\text { disturbances, previous neurodegenerative } \\
\text { disease, previous psychiatric disturbances, } \\
\text { dementia }\end{array}$ & No & $\begin{array}{l}\text { No left-sided } \\
\text { No right-sided } \\
\text { Frontal lobe }\end{array}$ & No & No & Yes & NS & NS \\
\hline
\end{tabular}


Table 1 (continued)

\begin{tabular}{|c|c|c|c|c|c|}
\hline Study & $\begin{array}{l}\mathrm{n}(\% \text { male }) \\
\text { Mean age }\end{array}$ & $\begin{array}{l}\text { Stroke type and lesion } \\
\text { location }\end{array}$ & Apathy & Apathy assessment & Study objectives \\
\hline $\begin{array}{l}\text { Mayo et al. } \\
2009[33]\end{array}$ & $\begin{array}{l}\text { First-ever } \\
408(59.1) \\
66.5[-]\end{array}$ & $\begin{array}{l}408 \text { (100) infarcts } \\
177 \text { (43.4) left; } \\
197 \text { (48.3) right } \\
18 \text { (4.4) bilateral }\end{array}$ & $82(20)$ & $\begin{array}{l}\text { Apathy index } \\
\text { Telephone } \\
\text { (caregivers) }\end{array}$ & $\begin{array}{l}\text { Apathy changes over the 1st year } \\
\text { after stroke and its impact on } \\
\text { recovery }\end{array}$ \\
\hline $\begin{array}{l}\text { Okada et al. } \\
1997[40]\end{array}$ & $\begin{array}{l}40(57.5) \\
71.4[-]\end{array}$ & 40 (100) subcortical infarcts & $20(50)$ & Apathy scale & $\begin{array}{l}\text { Relationship between apathy } \\
\text { and regional cerebral blood flow }\end{array}$ \\
\hline $\begin{array}{l}\text { Sagen et al. } \\
2010[22,23]\end{array}$ & $\begin{array}{l}104(59) \\
64.5[-]\end{array}$ & $\begin{array}{l}99 \text { (95.2) infarcts } \\
5 \text { (4.8) hemorrhages }\end{array}$ & $41(48.8)$ & Apathy evaluation scale & $\begin{array}{l}\text { Identify clinical predictors of } \\
\text { anxiety, depression and apathy } \\
\text { post-stroke }\end{array}$ \\
\hline $\begin{array}{l}\text { Withall et al. } \\
2010[3,24]\end{array}$ & $\begin{array}{l}\text { Case-control } \\
106(48.4) \\
74.9[-]\end{array}$ & 106 (100) infarcts & $27(25.5)$ & $\begin{array}{l}\text { Apathy evaluation scale - } \\
\text { informant }\end{array}$ & $\begin{array}{l}\text { Relationship between apathy } \\
\text { and depression longitudinally, } \\
\text { and its association with } \\
\text { dementia }\end{array}$ \\
\hline $\begin{array}{l}\text { Yamagata et al. } \\
2004[32]\end{array}$ & $\begin{array}{l}29(72.4) \\
71.7[56-87]\end{array}$ & $\begin{array}{l}29(100) \text { subcortical } \\
\text { infarcts } \\
11(37.9) \text { left; } 8 \text { (27.6) right } \\
10(34.5) \text { bilateral }\end{array}$ & $16(55.2)$ & Apathy scale & $\begin{array}{l}\text { Associations between apathy } \\
\text { and subcortical cerebral } \\
\text { infarctions }\end{array}$ \\
\hline \multicolumn{6}{|c|}{ Post-acute stage (>15 days): rehabilitation setting } \\
\hline $\begin{array}{l}\text { Castellanos-Pinedo et al. } \\
2011 \text { [41] }\end{array}$ & $\begin{array}{l}89(51.7) \\
-\quad[40-85]\end{array}$ & $\begin{array}{l}89 \text { (100) infarcts } \\
45 \text { (50.6) left; } 30 \text { (33.7) right }\end{array}$ & $31(34.8)$ & $\begin{array}{l}\text { Neuropsychiatric } \\
\text { inventory-apathy }\end{array}$ & $\begin{array}{l}\text { Identify clinical predictors of } \\
\text { psychopathological symptoms }\end{array}$ \\
\hline $\begin{array}{l}\text { Hama et al. } \\
2007[19,21]\end{array}$ & $\begin{array}{l}243(66.6) \\
65.2[-]\end{array}$ & $\begin{array}{l}\text { infarcts or hemorrhages [20] } \\
128(54) \text { infarcts } \\
109 \text { (46) hemorrhages }\end{array}$ & $98(40.3)$ & Apathy scale & $\begin{array}{l}\text { Correlation between stroke basal } \\
\text { ganglia lesion or frontal lobe } \\
\text { and depression }\end{array}$ \\
\hline $\begin{array}{l}\text { Santa et al. } \\
2008 \text { [35] }\end{array}$ & $\begin{array}{l}\text { First-ever } \\
67(56.7) \\
67.2[45-90]\end{array}$ & $\begin{array}{l}35 \text { (52.2) infarcts } \\
32 \text { (47.8) hemorrhages } \\
40 \text { (59.7) left; } 54(80.6) \text { right }\end{array}$ & $14(21)$ & Apathy scale & $\begin{array}{l}\text { Frequency of apathy after a } \\
\text { first-ever stroke and its impact } \\
\text { on functional recovery. }\end{array}$ \\
\hline
\end{tabular}

Data are expressed as percentages in parentheses or range in brackets. NS $=$ Not specified due to missing data in the original or secondary publication; SAH = subarachnoid hemorrhage; TIA = transient ischemic attack; ACA = anterior cerebral artery.

A total of 2,221 patients were evaluated in these 19 studies (range 29 [32] to 408 [33]). Ten of these studies evaluated more than 100 patients. All included patients with ischemic stroke and 7 studies also evaluated patients with intracerebral hemorrhages, in proportions ranging from 2 to $47.8 \%$ [5, 9, 19, 22, 34-36]. Among these 19 studies, 7 evaluated first-ever stroke patients (3 in acute stage and 4 in post-acute stage) $[9,27,33-35,37$, 38 ] and 12 included patients independently of prior history of cerebrovascular lesions $[3,5-7,19,22,26,32,36$, $39,40,41]$. The timing of apathy assessment after stroke varied across studies from 1 day to 15 months. Seven studies evaluated apathy in the acute stroke phase and 12 in the post-acute phase. Two studies reported the evolu- tion of apathy secondary to stroke over 1 year [33] or over a 15-month period [3]. With the exception of 3 studies that assessed patients in a rehabilitation setting $[19,35$, 41], all others were conducted in a hospital setting. Apathy was assessed using one of the following scales: Apathy Scale $(n=9)$ [42], Apathy Evaluation Scale $(n=4)[10$, 43], Neuropsychiatric Inventory $(n=2)$ [44], Emotion Behavior Index Form $(\mathrm{n}=1)$ [29] and Apathy Index Telephone $(n=1)$ [45]. In two studies [34, 39], apathy assessment was performed solely based on clinical examination or clinical interview-based questions without using any rating scale. Table 1 shows the main characteristics of included studies. 


\begin{tabular}{|c|c|c|c|c|c|c|c|}
\hline \multirow[t]{2}{*}{ Exclusion criteria } & \multicolumn{7}{|c|}{ Report of significant associations between apathy incidence and clinical factors } \\
\hline & stroke type & stroke location & age & gender & depression & $\begin{array}{l}\text { cognitive } \\
\text { impairment }\end{array}$ & $\begin{array}{l}\text { poor } \\
\text { outcome }\end{array}$ \\
\hline $\begin{array}{l}\text { Severe comorbidity, discharge to long-term } \\
\text { care }\end{array}$ & NS & NS & Yes & NS & Yes & Yes & Yes \\
\hline Aphasia, dementia or Alzheimer & NS & $\begin{array}{l}\text { Right } \\
\text { dorsolateral } \\
\text { frontal and left } \\
\text { fronto-temporal }\end{array}$ & No & No & Yes & Yes & No \\
\hline $\begin{array}{l}\text { TIA, aphasia, psychosis, severe cognitive } \\
\text { impairment, terminal illness }\end{array}$ & NS & NS & Yes & No & No & NS & No \\
\hline $\begin{array}{l}>85 \text { years old, hemorrhage, TIA, } \\
\text { consciousness, disturbance, non-English, } \\
\text { dementia, alcohol abuse, mental } \\
\text { retardation, aphasia }\end{array}$ & NS & NS & No & No & No & Yes & Yes \\
\hline Dementia & NS & $\begin{array}{l}\text { Frontal } \\
\text { (fronto- } \\
\text { subcortical } \\
\text { circuit) }\end{array}$ & No & No & No & Yes & NS \\
\hline $\begin{array}{l}\text { Dementia, hemorrhage, other brain } \\
\text { injuries, TIA, delirium }\end{array}$ & NS & Right-sided & No & No & No & No & Yes \\
\hline $\begin{array}{l}\text { Previous psychiatric disease, dementia or } \\
\text { aphasia, SAH }\end{array}$ & No & $\begin{array}{l}\text { Bilateral } \\
\text { No left-sided } \\
\text { No right-sided } \\
\text { Basal ganglia }\end{array}$ & Yes [21] & No [21] & No & No [21] & Yes [21] \\
\hline Aphasia, dementia & Infarct & $\begin{array}{l}\text { Left-sided } \\
\text { basal ganglia } \\
\text { No right-sided }\end{array}$ & Yes & No & NS & Yes & No \\
\hline
\end{tabular}

In terms of quality, of the 19 studies, $3(16 \%)$ were rated 'low', 11 (58\%) 'moderate' and 5 (26\%) 'high'. Most studies recruited a representative population (63.2\%), used explicit inclusion criteria (84.2\%) and consecutively recruited patients (84.2\%). However, it was only clear that patients entered the study at a similar stage of their disease and were prospectively recruited in 42.1 and $31.6 \%$ of the studies, respectively. Outcome assessment was properly made in $89.5 \%$ of the studies and almost all studies reported data for prognostic factors (94.7\%). The highest risk of study bias was found for follow-up. Only $26.3 \%$ of the studies reported and explained loss to follow-up or had a followup long enough for important events to occur. Details on quality assessment are provided in online suppl. fig. 1.

\section{Outcomes}

\section{Rate of Apathy}

Overall, the frequency of apathetic patients reported in individual studies ranged between 15.2 and $71.1 \%$. The pooled rate was $36.3 \%$ (95\% CI 30.3-42.8\%) with moderate heterogeneity $\left(\mathrm{I}^{2}=46.8 \%\right)$. Exclusion of the two studies $[34,39]$ that did not specify the use of validated scales for assessing apathy did not significantly change the pooled result $\left(37.0 \%\right.$; 95\% CI 30.5-43.9\%; $\left.\mathrm{I}^{2}=47.0 \%\right)$. Estimates were similar among studies in acute $[39.5 \%(95 \%$ CI $28.9-51.1 \%$; $\left.\left.\mathrm{I}^{2}=47.0 \%\right)\right]$ and post-acute $[34.3 \%(95 \%$ CI 27.8-41.4\%; $\mathrm{I}^{2}=45.7 \%$ )] stroke phases (subgroup difference: $4.7 \%$; $95 \% \mathrm{CI}-7.6$ to $17.1 \%$; $\mathrm{p}=0.455$; fig. $2 \mathrm{a}$ ). The 


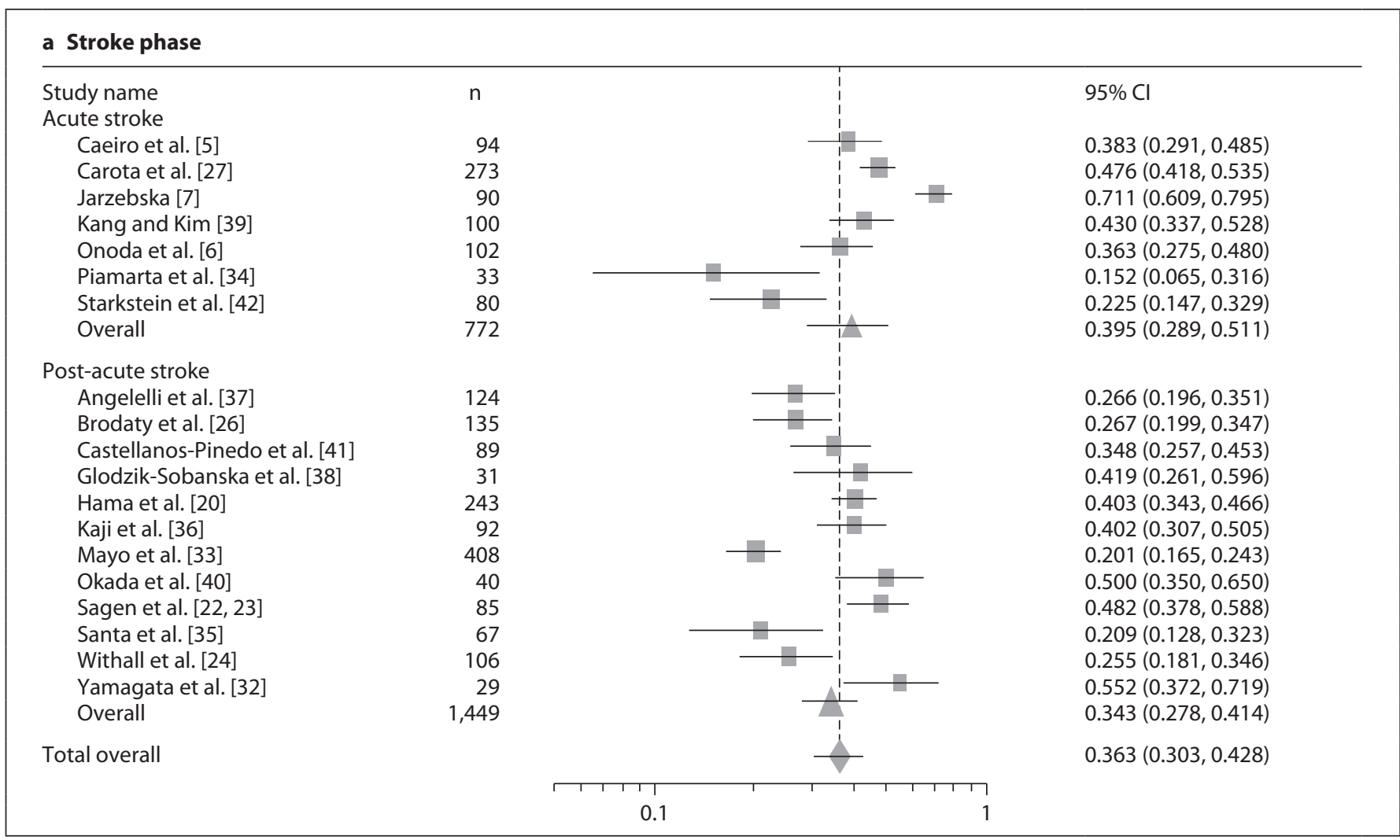

\section{b Stroke past history}

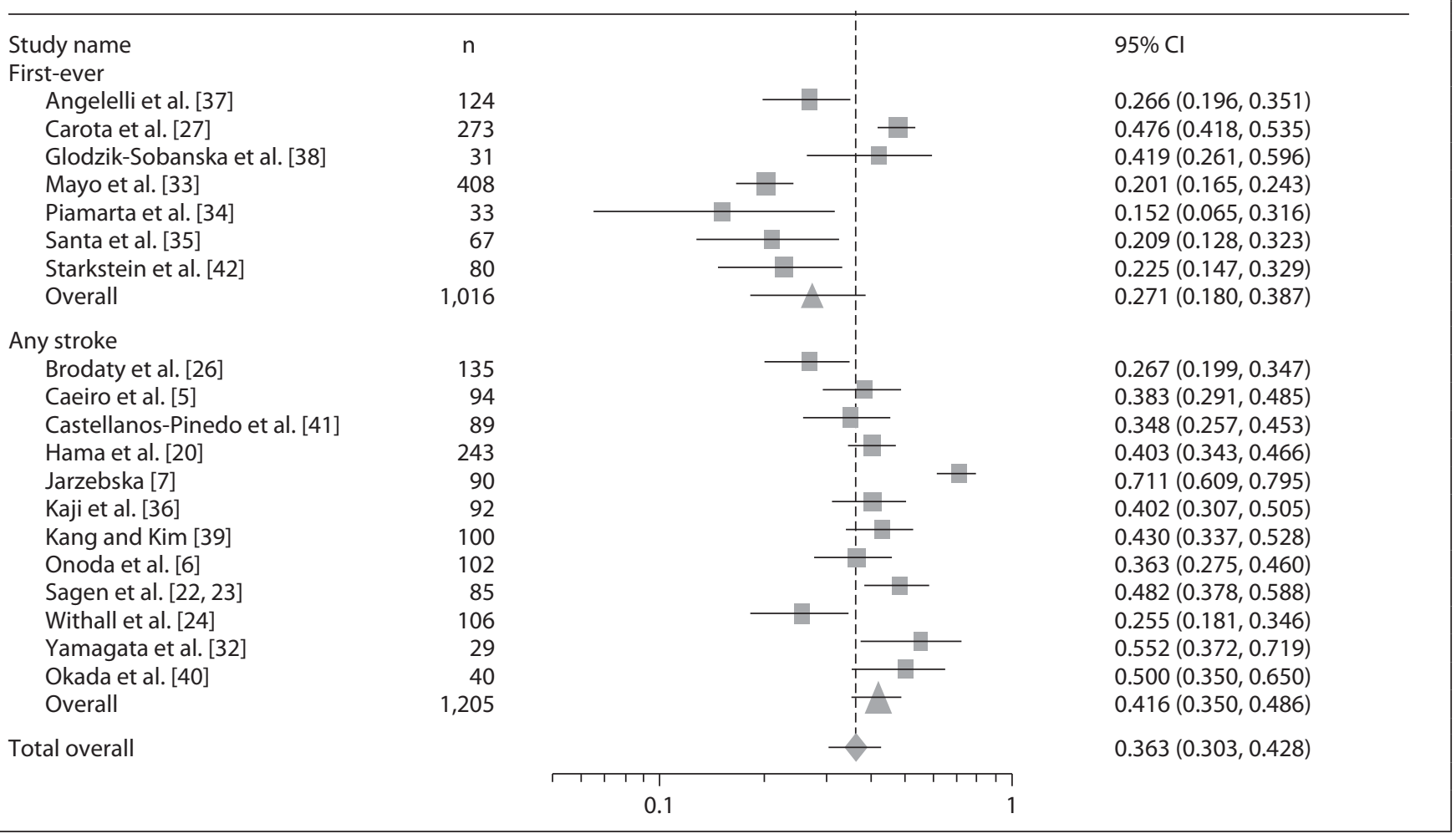




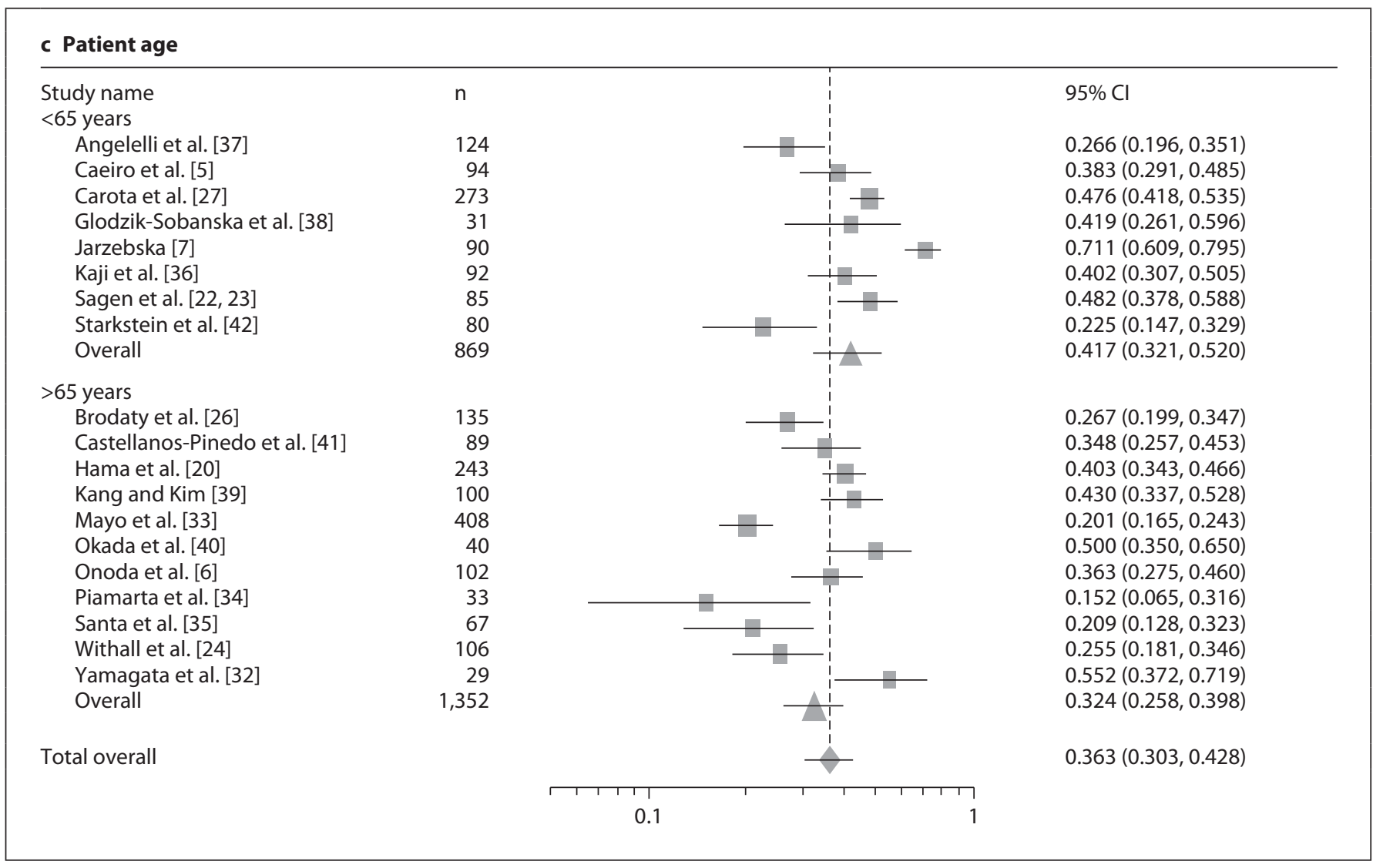

Fig. 2. a-c Rates of apathy.

apathy rate was significantly lower $(-14.3 \%$; $95 \%$ CI -2.2 to $-26.5 \%$; $\mathrm{p}=0.021$; fig. $2 \mathrm{~b}$ ) among first-ever stroke (27.1\%; 95\% CI $18.0-38.7 \%$; $\left.\mathrm{I}^{2}=47.7 \%\right)$ than any-stroke (41.6\%; 95\% CI 35.0-48.6\% I $\left.\mathrm{I}^{2}=44.9 \%\right)$ studies. The difference in apathy rate $(9.4 \%$; $95 \%$ CI -2.4 to $21.3 \%$; $\mathrm{p}=$ 0.118 ; fig. 2c) between studies evaluating younger (41.7\%; 95\% CI 32.1-52.0\%; $\left.\mathrm{I}^{2}=46.7 \%\right)$ and older patients (32.4\%; 95\% CI 25.8-39.8\%; $\left.\mathrm{I}^{2}=45.7 \%\right)$ was not significant. Metaregression also failed to show a significant relationship between apathy rate and patients mean age $(95 \%$ CI for regression coefficient: -0.046 to $0.006 ; \mathrm{p}=0.140$; online suppl. fig. 2).

The rate of apathy without concomitant depression was reported in 9 studies with a pooled estimate of $21.4 \%$ (95\% CI $\left.15.6-28.7 \% ; \mathrm{I}^{2}=45.6 \%\right)$. This rate was also lower in first-ever studies $(-15.6 \%$; $95 \%$ CI -4.9 to $-26.3 \%$; $\mathrm{p}=$ 0.004 ) and similar between acute vs. post-acute and be- tween younger vs. older patients ( $p>0.83$ for both comparisons). Figure $2 \mathrm{~d}-\mathrm{f}$ shows the main results for apathy rate without concomitant depression.

Two studies provided longitudinal data for apathy rate. In one study [3], about $55 \%$ of the patients who were apathetic at 4 months remained apathetic (with or without concomitant depression) at 15 months. In this study, dementia was found to be a risk factor for apathy. In the other longitudinal study [33], serial measures of apathy over time (12 months) were taken to estimate the extent of each apathy change. According to the authors, the majority $(50 \%)$ of the patients demonstrated apathetic behavior rarely and were classified as 'low-apathy'. In about one-third of the patients, the extent of apathetic behavior remained stable, while in the others, apathy either increased (7\%) or improved (7\%) over time. Older age, cognitive impairment and functional disability were found 


\section{d Stroke phase}

Study name

Acute stroke

Starkstein et al. [42]

Caeiro et al. [5]

Overall

Post-acute stroke

Brodaty et al. [26]

Hama et al. [19-21]

Mayo et al. [33]

Okada et al. [40]

Santa et al. [35]

Withall et al. [24]

Yamagata et al. [32

Overall

80

94

174

243

408

40

67

106

29

1,026
Total overall

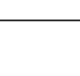

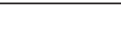

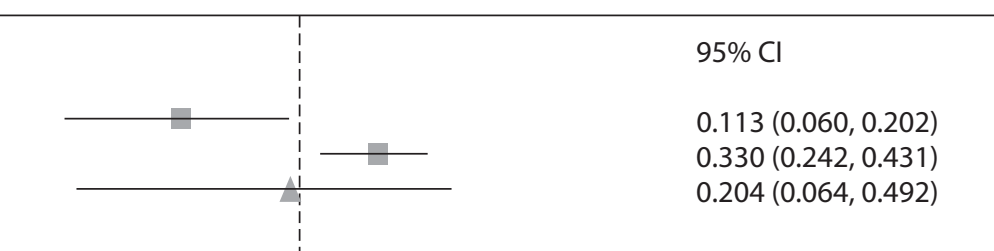

135

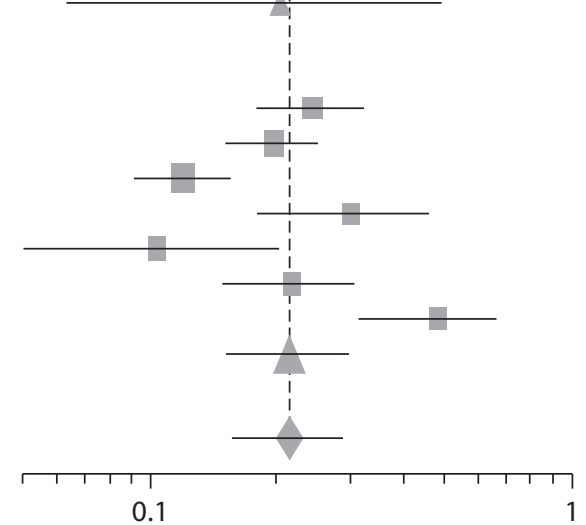

$0.244(0.179,0.324)$

$0.198(0.152,0.252)$

$0.120(0.092,0.155)$

$0.300(0.179,0.457)$

$0.104(0.051,0.203)$

$0.217(0.149,0.305)$

$0.483(0.311,0.659)$

$0.215(0.152,0.295)$

$0.214(0.156,0.287)$

\section{1}

\section{e Stroke past history}

\section{Study name}

First-ever

Mayo et al. [33]

Santa et al. [35]

Starkstein et al. [42]

Overall

Any stroke

Brodaty et al. [26]

Caeiro et al. [5]

Hama et al. [19-21]

Okada et al. [40]

Withall et al. [24]

Yamagata et al. [32]

Overall

Total overall

$n$
408
67
80
555

$\mathrm{n}$

408

67

555

135

94

243

40

106

29

647
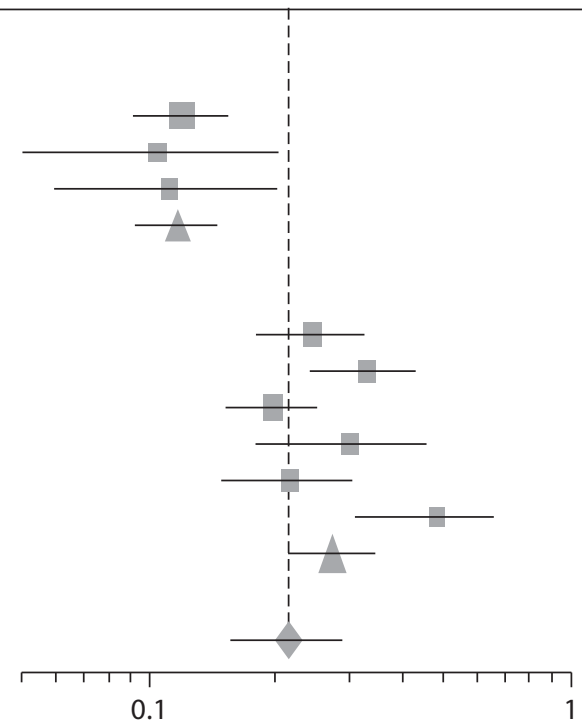

$95 \% \mathrm{Cl}$

$0.120(0.092,0.155)$

$0.104(0.051,0.203)$

$0.113(0.060,0.202)$

$0.117(0.093,0.147)$

$0.244(0.179,0.324)$

$0.330(0.242,0.431)$

$0.198(0.152,0.252)$

$0.300(0.179,0.457)$

$0.217(0.149,0.305)$

$0.483(0.311,0.659)$

$0.273(0.212,0.345)$

$0.214(0.156,0.287)$ 


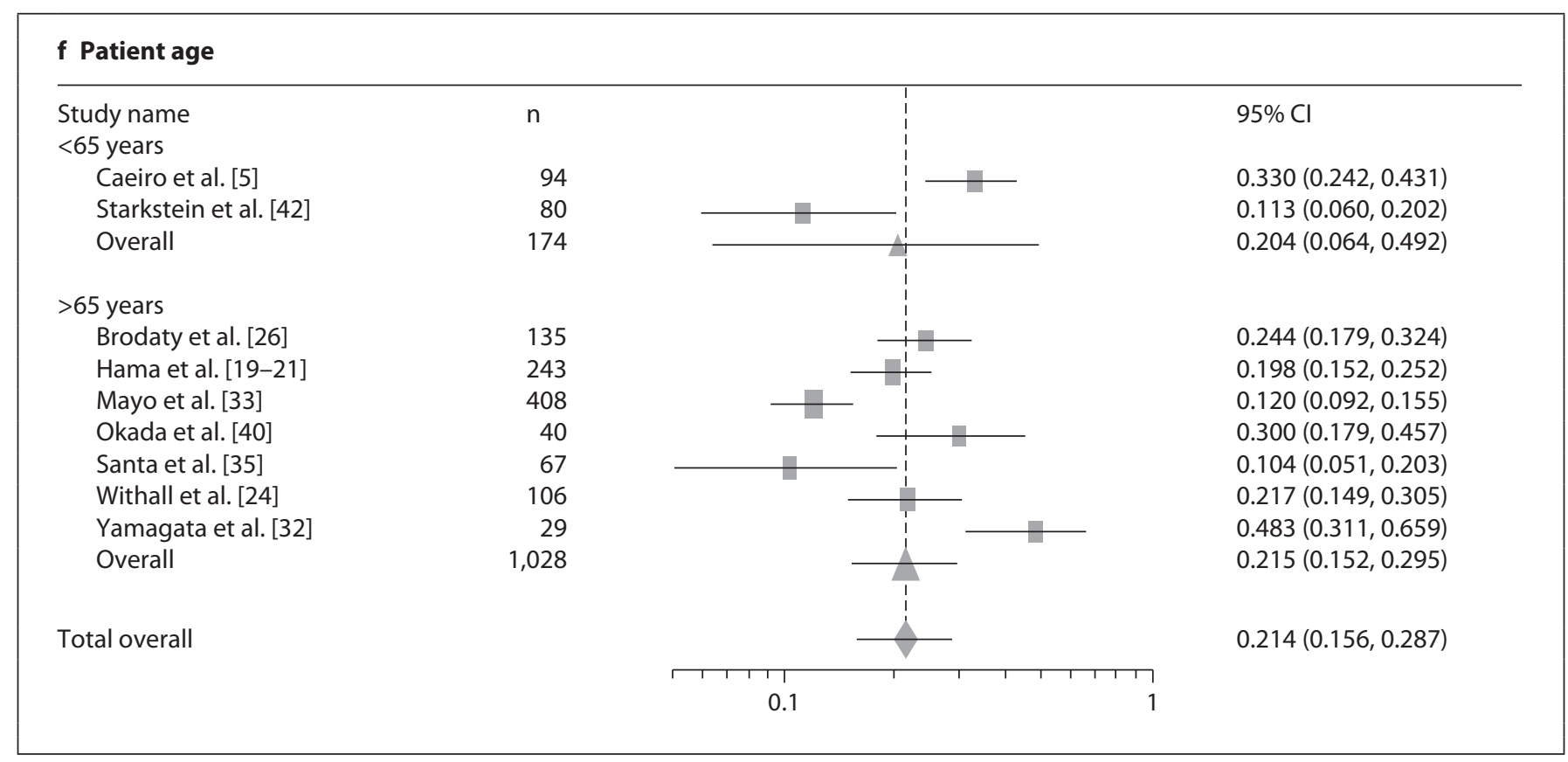

Fig. 2. d-f Apathy rate (without concomitant depression).

to be predictors of apathy after stroke. In this study, 'highapathy' had a significant negative effect on clinical outcomes.

\section{Apathy and Associated Factors}

Overall, apathetic patients were about 3 years older than nonapathetic patients (2.74 years; $95 \%$ CI 1.25-4.23; $\mathrm{I}^{2}=0 \%$ ). The proportion of females (OR 1.07; 95\% CI $0.80-1.42 ; \mathrm{I}^{2}=0 \%$ ) and males (OR 0.88; 95\% CI 0.66-1.17; $\left.\mathrm{I}^{2}=0 \%\right)$ was similar among apathetic and nonapathetic patients (fig. 3a).

The rate of apathy was similar in patients with left- and right-sided hemispheric lesions (OR 1.14; 95\% CI 0.602.15; fig. 3b). However, significant heterogeneity existed between the studies' results $\left(\mathrm{I}^{2}=63 \%\right)$. Although similar results were found for acute and post-acute stroke phase studies, no heterogeneity existed for post-acute stroke results. On the other hand, pooled results for studies evaluating older patients showed a significantly higher rate of apathy in left-sided lesions, without heterogeneity (OR 2.13 ; 95\% CI 1.19-3.80; $\left.\mathrm{I}^{2}=0 \%\right)$. Results were similar for first-ever and any-stroke, and both had significant heterogeneity (online suppl. fig. 3a).

Apathy Secondary to Stroke: A Systematic Review
Only three studies provided data for the analysis of the association between the rate of apathy and stroke type, which precludes strong conclusions. Overall, rate of apathy was similar between patients with hemorrhagic and ischemic strokes (OR 1.16; 95\% CI 0.25-5.26; fig. 3b). The high heterogeneity $\left(\mathrm{I}^{2}=79 \%\right)$ could at least partly be explained by the stroke phase and patient age. Two out of the three studies that provided data for this outcome evaluated younger patients in an acute stroke phase. In pooled results from these two studies, apathy was more frequent after hemorrhagic than ischemic stroke (OR 2.58; 95\% CI: $1.18-5.65 ; \mathrm{I}^{2}=0 \%$; online suppl. fig. $3 \mathrm{~b}$ ), while in older post-acute stroke patients apathy was less frequent after hemorrhagic stroke (OR 0.23; 95\% CI 0.06-0.90). Past history of stroke did not explain heterogeneity and no significant differences existed between first-ever and any-stroke studies.

The overall rate of depression without concomitant apathy found in included studies was $12.1 \%$ (95\% CI 8.2$\left.17.3 ; \mathrm{I}^{2}=43.4 \%\right)$. Depression was more common in apathetic than in nonapathetic patients (OR 2.29; 95\% CI $1.41-3.72$; $\mathrm{I}^{2}=44 \%$; fig. $3 \mathrm{c}$ ). Depression severity, as assessed through validated scales (Montgomery and As-

Cerebrovasc Dis 2013;35:23-39 


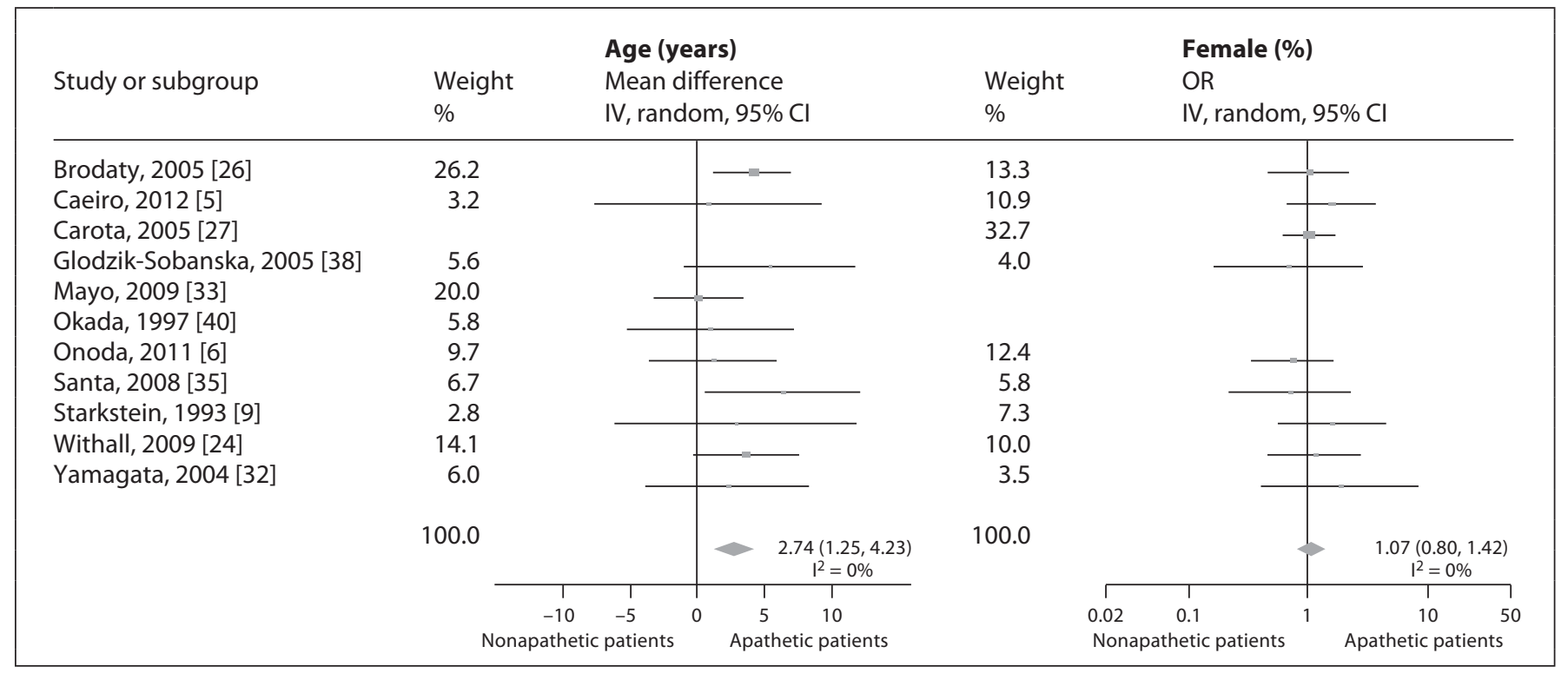

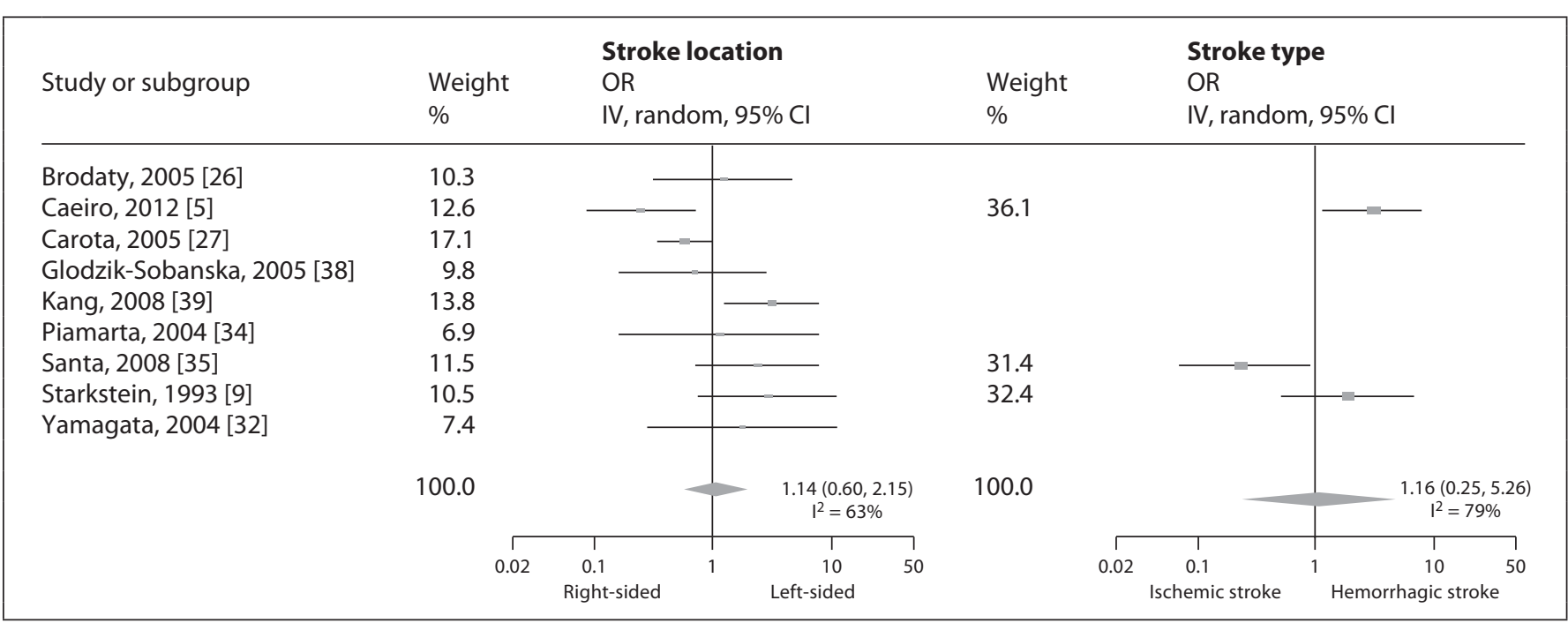

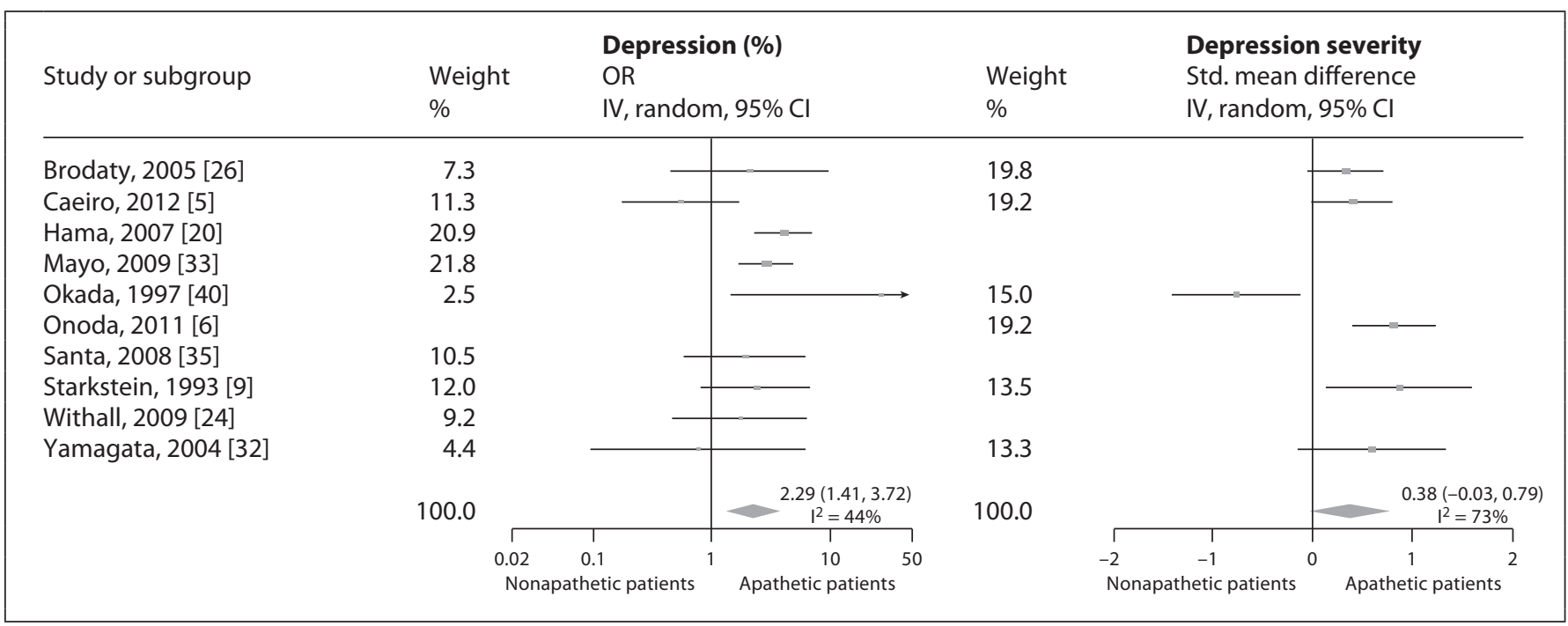




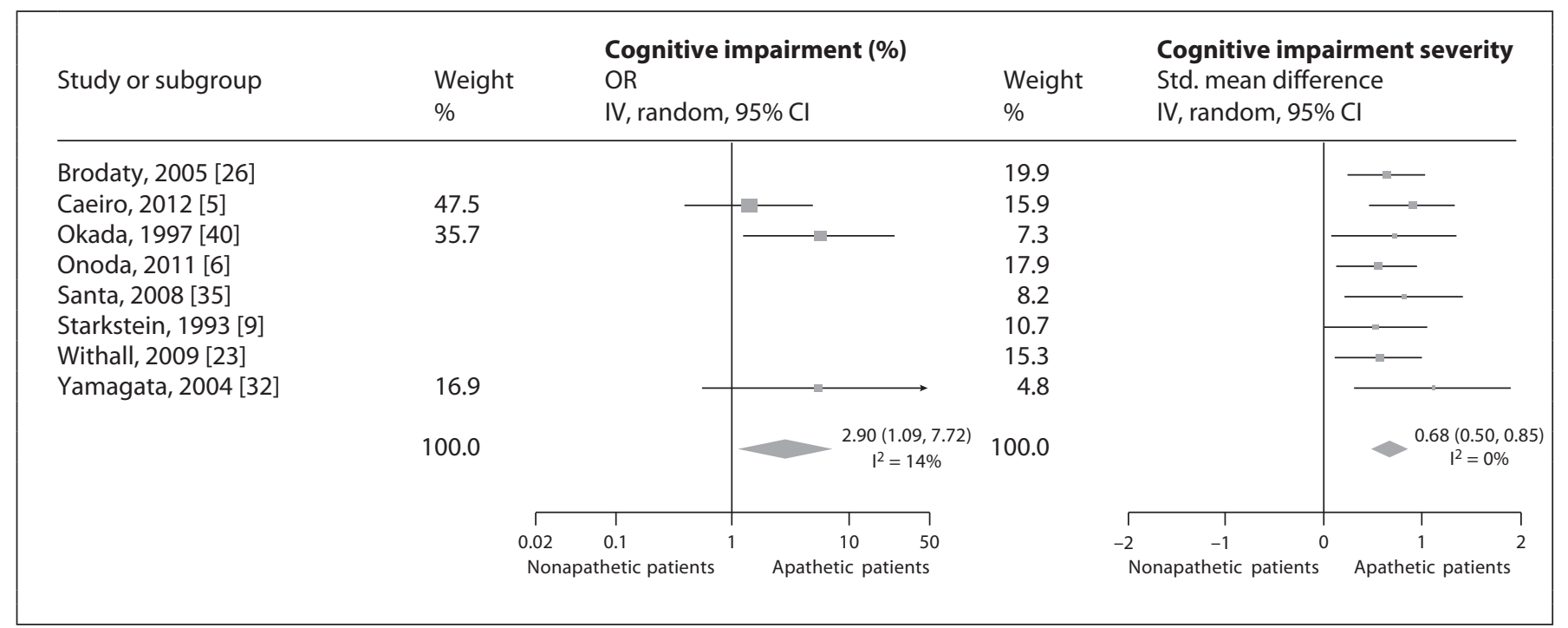

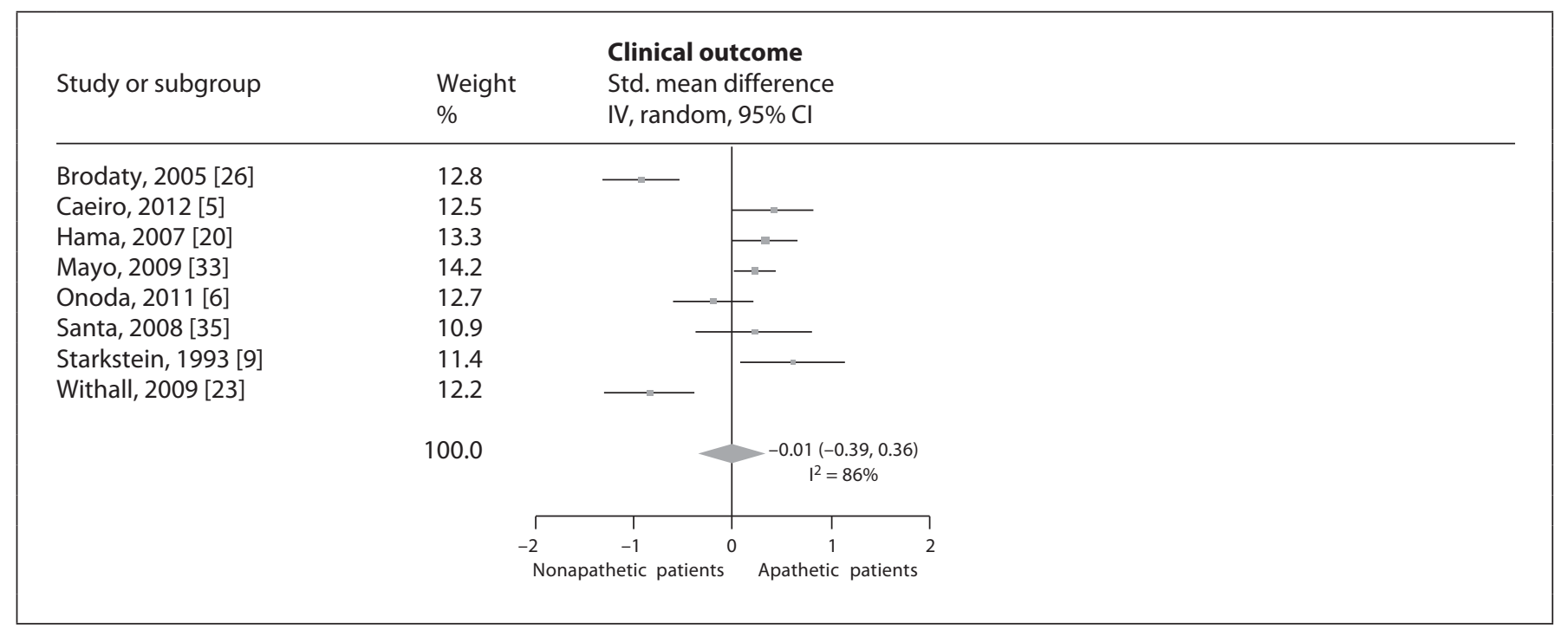

Fig. 3. a-e Apathy and associated factors.

berg Depression Rating Scale, Hamilton Depression Rating Scale and Self-Rating Depression Scale), was higher in apathetic patients (SMD 0.38; 95\% CI -0.03-0.79; $\mathrm{I}^{2}=$ $73 \%)$, although it did not reach significance ( $\mathrm{p}=0.07$; fig. 3c). The high heterogeneity $\left(\mathrm{I}^{2}=73 \%\right)$ could at least partly be explained by the stroke phase and patient age. Pooled results from the three acute-phase studies (SMD 0.65; 95\% CI 0.35-0.95) and the two studies evaluating younger patients (SMD 0.53; 95\% CI 0.12-0.94) showed a significantly higher depression severity with low heterogeneity $\left(\mathrm{I}^{2}=13 \%\right.$; online suppl. fig. $\left.3 \mathrm{c}\right)$.

The overall rate of patients with cognitive impairment found in included studies was 20.2\% (95\% CI 10.1-36.5; $\left.\mathrm{I}^{2}=42.5 \%\right)$. Cognitive impairment was more common in apathetic than in nonapathetic patients (OR 2.90; 95\% CI $1.09-7.72 ; \mathrm{I}^{2}=14 \%$ ), although this estimate is based on data from only 3 studies (fig. 3d). Severity of cognitive impairment, as assessed through Mini-Mental State Ex- 
amination and Hasegawa Dementia Rating Scale Revised, was higher in apathetic patients $(\mathrm{SMD}=0.68 ; 95 \%$ CI $0.50-0.85 ; \mathrm{I}^{2}=0 \%$; fig. $3 \mathrm{~d}$ ).

The severity of clinical global outcome, as assessed through validated scales (Modified Ranking Scale, Johns Hopkins Functioning Inventory, Instrumental Activities of Daily Living, Functional Independence Measurement and Barthel Index), was not significantly different between apathetic and nonapathetic patients (SMD -0.01; $95 \%$ CI -0.39 to 0.36 ), although significant heterogeneity $\left(\mathrm{I}^{2}=86 \%\right)$ exists among studies (fig. $3 \mathrm{e}$ ). The high heterogeneity could at least partly be explained by the stroke past history and patient age. Pooled results from the three first-ever stroke studies (SMD 0.29; 95\% CI 0.10-0.48) and the two studies evaluating younger patients (SMD 0.53 ; 95\% CI 0.12-0.94) showed a significant poorer clinical outcome in these apathetic patients with low heterogeneity ( $\mathrm{I}^{2}=0$ to $13 \%$; online suppl. fig. $3 \mathrm{~d}$ ).

In general, the results for the different outcomes did not change after excluding the studies rated as 'low quality' from the analysis including the two studies $[34,39]$ that evaluated apathy through clinical observation without specifying the use of validated scales (sensitivity analysis). The only two exceptions were depression severity which became significantly worse in apathetic patients (SMD 0.55; 95\% CI 0.28-0.81) and the rate of cognitive impairment which became nonsignificant (OR 1.4; 95\% CI 0.39-4.96).

\section{Discussion}

Apathy is thought to be a frequent complication of stroke associated with poorer outcomes, but available clinical data lacks strength and published results have been contradictory. We performed a systematic review and meta-analysis of all studies evaluating apathy secondary to stroke to better estimate its rate and risk factors, and explore associations with poorer outcomes. The most relevant findings of our study are: (1) apathy secondary to stroke is a frequent neuropsychiatric disturbance affecting 1 in every 3 stroke patients; apathy rate is lower in patients without previous cerebrovascular disease; the rate of 'pure' apathy (without concomitant depression) is twice as frequent as the rate of 'pure' depression (without concomitant apathy); (2) apathetic patients are about 3 years older than nonapathetic patients without gender differences; (3) rate of apathy was similar for left- and right-sided hemispheric stroke lesions and for ischemic and hemorrhagic stroke type; (4) apathetic pa- tients are more frequently and severely depressed and cognitively impaired in comparison to nonapathetic patients, and (5) overall, apathy secondary to stroke does not appear to have a negative impact on clinical global outcome, except for apathetic patients with first-ever stroke and for younger patients.

Our conclusions are based on 19 studies that evaluated apathy secondary to stroke ( 7 in acute phase and 12 in post-acute phase) enrolling a total of 2,221 patients. The overall quality of the studies was considered to be moderate. The highest risk of bias was found for follow-up (duration, reporting and explanation of lost cases). In most of the studies it is unclear whether patients were prospectively recruited or not. These aspects increase the risks of detection and selective reporting. However, sensitivity analysis by excluding studies rated 'low quality' did not significantly change the overall results.

We found high statistical heterogeneity $\left(\mathrm{I}^{2} \geq 50 \%\right)$ among the studies' results for stroke lesion lateralization and type and for depression and clinical global outcome severity, but not for the other outcomes. The heterogeneity found in these outcomes was most probably due to differences in stroke phase (acute and post-acute phase) and history (first-ever and any-stroke), patient age (younger and older) and study quality. In fact, heterogeneity decreases when considering the results for each stroke subgroup separately and after excluding the studies rated as low quality from the analysis.

We were conservative in our analysis because we did not consider undefined data, and when studies presented different estimates we extracted those reporting only the most precise or adjusted measure. We were also able to retrieve unpublished information from two studies. Nevertheless, moderate heterogeneity exists among studies in our primary outcome.

The rates of apathy secondary to stroke found in our study are undoubtedly large, which could be due to the absence of a clear nosological definition or clinical criteria $[46,47]$. However, the mean ages of included samples (range 53-75 years) were lower than that usually reported in hospital-based stroke samples, which could indicate the presence of selection bias in the included studies and result in an underestimate of apathy rate. In the two studies that provided longitudinal data [3,33], about half of the apathetic patients remained apathetic long after stroke onset and most of these cases rarely had apathetic behaviors. Older age, cognitive impairment and functional disability were found to be predictors of apathy after stroke. 
According to our results, apathetic patients were older and more frequently and severely cognitively impaired. These results are in agreement with recent studies of prospective healthy community-dwelling populations [48, 49]. Similar to the results from longitudinal post-stroke studies $[3,33]$, in these studies the frequency of apathy increased with age and was positively correlated with the presence and severity of cognitive impairment. Furthermore, apathy was associated with cognitive and functional impairments in elders adjudged to have normal cognition [48]. It has been reported that up to a quarter of nondemented community-dwelling elderly individuals have apathy (most without concomitant depression) [50, 51]. In this population a past history of stroke (OR 1.8; 95\% CI 1.4-2.3) [50] or other vascular diseases are risk factors for apathy [51]. The prevalence of apathy is significantly higher in demented patients [52]. A prospective multicenter study evaluating 684 community-dwelling elderly patients with Alzheimer disease reported an apathy point prevalence of $43 \%$, which was associated with functional disability [53]. Other studies in community-dwelling elderly patients with dementia have reported even higher apathy prevalence rates [54]. Similar to nondemented older people, a previous history of stroke also increases the risk of apathy (OR 4.5; 95\% CI 1.3-15.6) in older demented people [55]. In summary, apathy is a common condition in older individuals and in particular in older cognitively impaired people. Stroke is a risk factor for apathy in both.

In a review with five studies, Jorge et al. [56] drew attention to the fact that apathy is often associated with depression, but one can occur separately from the other. We found that the rate of apathy without depression was twice that of depression without apathy, further reinforcing the notion that these are different clinical entities. On the other hand, our results also show an association between apathy and depression, as apathetic patients are more frequently depressed and apathy rate increases 15\% in the presence of depression. In a prospective study that specifically evaluated the relationship between apathy and depression following stroke, no significant overlap between both syndromes were found at index assessment [3]. However, apathy and depression overlap longitudinally 1 year after stroke, possibly indicating that cumulative vascular lesions are an important risk factor for both. Furthermore, dementia was a common risk factor for both syndromes in stroke patients.

Apathy and depression share symptoms such as diminishing interest in daily activities and decrease in motion activity. Patients with both neuropsychiatric distur-

Apathy Secondary to Stroke: A Systematic Review bances after stroke share common neuropsychological features such as low MMSE scores [3] and working memory impairment [26]. Specific subcortical stroke locations can induce both apathy and depression $[3,26]$.

We found no differences in the rate of apathy according to the hemispheric stroke side location, except for older ages, which have apathy more frequently after left-sided stroke lesions. Jorge et al. [56] suggested that stroke involving subcortical areas of the cortico-subcortical circuits is associated with apathy. Specific stroke locations have been reported to be related to apathy, namely basal ganglia lesions (leading to a dysfunction of the frontosubcortical system) [6], with possible involvement of the dopaminergic and glutamatergic systems [38, 57], anterior thalamic acute lesions [58, 59], polar-paramedian thalamic lesions [57] and amygdala lesions [25]. Abulia in the post-acute stroke phase was found in $55 \%$ of patients with caudate stroke lesions [60]. However, these statements are based on reports investigating apathy in patients with specific focal stroke location without controls. We have not included these studies in our analysis in order to decrease selection bias.

Although some publications have suggested the existence of an association between apathy secondary to stroke and a lower functional status [56], our results do not confirm those claims, except for samples including first-ever stroke and younger patients. The apparent discrepancy of our finding may be related to characteristics of apathy itself because apathetic patients may be less aware of or report fewer complaints about a loss of functionality.

Our systematic review has several limitations. The results and conclusion are weakened by limitations inherent to meta-analysis and individual studies. Except for the rate of apathy (our primary outcome), a significant number of publications did not report data for other outcomes, in particular for the relationship between apathy and associated factors. Our analysis included only institution-based studies. Although our results are in close agreement with those from prospective communitydwelling population studies, they should be generalized with caution to the overall post-stroke population. Furthermore, studies also used different scales to evaluate apathy, depression, cognitive impairment and clinical global outcome. None of the studies reported information about stroke severity, which may have had a negative impact on motivational status. The overall quality of included studies was moderate. However, reporting quality for a few studies was low and selective and detection bias cannot be ruled out. The exclusion of publications writ- 
ten in languages other than English, German, Spanish, Portuguese or French, as well as of nonpublished studies, may increase the risk of publication bias. Nevertheless, we were able to obtain nonpublished data from two studies and visual inspection of funnel plots shows symmetry, suggesting that publication bias was not a major drawback of our review.

In conclusion, in hospital and rehabilitation-based studies apathy secondary to stroke is a common neuropsychiatric disturbance both in acute and post-acute phases. Future research is needed to properly address how apathy and depression are related and affect cognitive functioning, specifically executive functioning. Representative prospective cohort-studies with diffusion- weighted MR imaging that properly evaluate longitudinally apathy and its association with stroke type and severity, as well as with concomitant outcomes, are strongly needed. The high rate of post-stroke apathy should also prompt the evaluation of therapeutic options for apathy treatment.

\section{Acknowledgements}

This review was partly supported by the Fundação para a Ciência e a Tecnologia, from the $\mathrm{PhD}$ scholarship (ref.: SFRH/ BD/22282/2005) attributed to Lara Caeiro.

We thank the Portuguese Cochrane Collaborating Center for technical support in the conduction of this systematic review.

\section{References}

1 Marin RS: Apathy: a neuropsychiatric syndrome. J Neuropsychiatry Clin Neurosci 1991;3:243-254.

2 Marin RS: Differential diagnosis and classification of apathy. Am J Psychiatry 1990;147: $22-30$.

3 Withall A, Brodaty H, Altendorf A, Sachdev PS: A longitudinal study examining the independence of apathy and depression after stroke: the Sydney Stroke Study. Int Psychogeriatr 2011;23:264-273.

4 Singh M, Cameron J: Psychosocial aspects of caregiving to stroke patients. Axone 2005;27: $18-24$.

$\checkmark 5$ Caeiro L, Ferro JM, Figueira ML: Apathy in acute stroke patients. Eur J Neurol 2012;19: 291-297.

6 Onoda K, Kuroda Y, Yamamoto Y, Abe S, Oguro H, Nagai A, Bokura H, Yamaguchi S: Post-stroke apathy and hypoperfusion in basal ganglia: SPECT study. Cerebrovasc Dis 2011;31:6-11.

-7 Jarzebska E: Stroke patients' apathy (in Polish). Pol Merkur Lekarski 2007;22:280-282.

-8 Stroup DF, Berlin JA, Morton SC, Olkin I, Williamson GD, Rennie D, Moher D, Becker BJ, Sipe TA, Thacker SB: Meta-analysis of observational studies in epidemiology: a proposal for reporting - Meta-Analysis Of Observational Studies in Epidemiology (MOOSE) group. JAMA 2000;283:20082012.

-9 Starkstein SE, Fedoroff JP, Price TR, Leiguarda R, Robinson RG: Apathy following cerebrovascular lesions. Stroke 1993;24: 1625-1630.

10 Marin RS, Biedrzycki RC, Firinciogullari S: Reliability and validity of the Apathy Evaluation Scale. Psychiatry Res 1991;38:143-162.
11 Dalziel K, Round A, Stein K, Garside R, Castelnuovo E, Payne L: Do the findings of case series studies vary significantly according to methodological characteristics? Health Technol Assess 2005;9:iii-iv, 1-146.

12 Chambers D, Rodgers M, Woolacott N: Not only randomized controlled trials, but also case series should be considered in systematic reviews of rapidly developing technologies. J Clin Epidemiol 2009;62:1253-1260.

13 Khan KS: Undertaking Systematic Reviews of Research on Effectiveness: CRD's Guidance for Those Carrying Out or Commissioning Reviews, ed 2. York, NHS Centre for Reviews and Dissemination, 2001.

14 Wallace BC, Schmid CH, Lau J, Trikalinos TA: Meta-analyst: software for meta-analysis of binary, continuous and diagnostic data. BMC Med Res Methodol 2009;9:80.

15 Higgins JPT, Green S: Cochrane Handbook for Systematic Reviews of Interventions Version 5.1.0. Chichester, Wiley \& Sons, 2011.

16 Khan KS, Kinz R, Kleijnen J, Antes G: Systematic reviews to support evidence-based medicine. London: Royal Society of Medicine Press, 2003.

17 Deeks JJ Altman DG, Bradburn MJ: Statistical methods for examining heterogeneity and combining results from several studies in metaanalysis. London, BMJ Publication Group, 2001, pp 313-335.

18 Deeks JJ: Issues in the selection of a summary statistic for meta-analysis of clinical trials with binary outcomes. Stat Med 2002;21: $1575-1600$.

19 Hama S, Yamashita H, Shigenobu M, Watanabe A, Kurisu K, Yamawaki S, Kitaoka T: Post-stroke affective or apathetic depression and lesion location: left frontal lobe and bilateral basal ganglia. Eur Arch Psychiatry Clin Neurosci 2007;257:149-152.
20 Hama S, Yamashita H, Shigenobu M, Watanabe A, Hiramoto K, Kurisu K, Yamawaki S, Kitaoka T: Depression or apathy and functional recovery after stroke. Int J Geriatr Psychiatry 2007;22:1046-1051.

21 Hama S, Yamashita H, Shigenobu M, Watanabe A, Hiramoto K, Takimoto Y, Arakawa R, Kurisu K, Yamawaki S, Kitaoka T: Sitting balance as an early predictor of functional improvement in association with depressive symptoms in stroke patients. Psychiatry Clin Neurosci 2007;61:543-551.

22 Sagen U, Finset A, Moum T, Mørland T, Vik TG, Nagy T, Dammen T: Early detection of patients at risk for anxiety, depression and apathy after stroke. Gen Hosp Psychiatry 2010;32:80-85

23 Sagen U, Faerden A, Haug T, Melle I, Finset A, Dammen T: Are there common core features of apathy in different neuropsychiatric samples as assessed by the Apathy Evaluation Scale? Nord J Psychiatry 2010;64: 49-57.

24 Withall A, Brodaty H, Altendorf A, Sachdev PS: Who does well after a stroke? The Sydney stroke study. Aging Ment Health 2009;13: 693-698.

25 Sachdev PS, Chen X, Joscelyne A, Wen W, Brodaty H: Amygdala in stroke/transient ischemic attack patients and its relationship to cognitive impairment and psychopathology: the Sydney Stroke Study. Am J Geriatr Psychiatry 2007;15:487-496.

-26 Brodaty H, Sachdev PS, Withall A, Altendorf A, Valenzuela MJ, Lorentz L: Frequency and clinical, neuropsychological and neuroimaging correlates of apathy following stroke - the Sydney Stroke Study. Psychol Med 2005;35:1707-1716. 
-27 Carota A, Berney A, Aybek S, Iaria G, Staub F, Ghika-Schmid F, Annable L, Guex P, Bogousslavsky J: A prospective study of predictors of poststroke depression. Neurology 2005;64:428-433.

28 Aybek S, Carota A, Ghika-Schmid F, Berney A, Melle GV, Guex P, Bogousslavsky J: Emotional behavior in acute stroke: the Lausanne emotion in stroke study. Cogn Behav Neurol 2005;18:37-44.

29 Ghika-Schmid F, van Melle G, Guex P, Bogousslavsky J: Subjective experience and behavior in acute stroke: the Lausanne Emotion in Acute Stroke Study. Neurology 1999; 52:22-28.

-30 Caeiro L, Ferro JM, Santos CO, Figueira ML: Depression in acute stroke. J Psychiatry Neurosci 2006;31:377-383.

- 31 Marasco G, Iavarone A, Ronga B, Martini V, Crispino M, Postiglione A: Depressive symptoms in patients admitted to a semi-intensive stroke unit. Acta Neurol Belg 2011;111:276281.

- 32 Yamagata S, Yamaguchi S, Kobayashi S: Impaired novelty processing in apathy after subcortical stroke. Stroke 2004;35:19351940.

- 33 Mayo NE, Fellows LK, Scott SC, Cameron J, Wood-Dauphinee S: A longitudinal view of apathy and its impact after stroke. Stroke 2009;40:3299-3307.

- 34 Piamarta F, Iurlaro S, Isella V, Atzeni L, Grimaldi M, Russo A, Forapani E, Appollonio I: Unconventional affective symptoms and executive functions after stroke in the elderly. Arch Gerontol Geriatr 2004;9:315323.

- 35 Santa N, Sugimori H, Kusuda K, Yamashita Y, Ibayashi S, Iida M: Apathy and functional recovery following first-ever stroke. Int J Rehabil Res 2008;31:321-326.

- 36 Kaji Y, Hirata K, Ebata A: Characteristics of post-stroke depression in Japanese patients. Neuropsychobiology 2006;53:148-152.

- 37 Angelelli P, Paolucci S, Bivona U, Piccardi L, Ciurli P, Cantagallo A, Antonucci G, Fasotti L, Di Santantonio A, Grasso MG, Pizzamiglio L: Development of neuropsychiatric symptoms in poststroke patients: a crosssectional study. Acta Psychiatr Scand 2004; 110:55-63.

- 38 Glodzik-Sobanska L, Slowik A, Kieltyka A, Kozub J, Sobiecka B, Urbanik A, Szczudlik A: Reduced prefrontal $\mathrm{N}$-acetylaspartate in stroke patients with apathy. J Neurol Sci 2005;238:19-24.
-39 Kang SY, Kim JS: Anterior cerebral artery infarction: stroke mechanism and clinical-imaging study in 100 patients. Neurology 2008; 70:2386-2393.

40 Okada K, Kobayashi S, Yamagata S, Takahashi K, Yamaguchi S: Poststroke apathy and regional cerebral blood flow. Stroke 1997;28: 2437-2441.

41 Castellanos-Pinedo F, Hernández-Pérez JM, Zurdo M, Rodríguez-Fúnez B, HernándezBayo JM, García-Fernández C, Cueli-Rincón B, Castro-Posada JA: Influence of premorbid psychopathology and lesion location on affective and behavioral disorders after ischemic stroke. J Neuropsychiatry Clin Neurosci 2011;23:340-347.

42 Starkstein SE, Mayberg HS, Preziosi TJ, Andrezejewski P, Leiguarda R, Robinson RG: Reliability, validity, and clinical correlates of apathy in Parkinson's disease. J Neuropsychiatry Clin Neurosci 1992;4:134-139.

43 Caeiro L, Ferro JM: Validation of a short form of the Apathy Evaluation Scale-Clinical version. Cerebrovasc Dis 2008;25(suppl. 2):187.

44 Cummings JL, Mega M, Gray K, RosenbergThompson S, Carusi DA, Gornbein J: The Neuropsychiatric Inventory: comprehensive assessment of psychopathology in dementia. Neurology 1994;44:2308-2314.

-45 Cameron JI, Cheung AM, Streiner DL, Coyte PC, Singh MD, Stewart DE: Factor structure and reliability of the brain impairment behavior scale. J Neurosci Nurs 2008;40:40-47.

46 American Psychiatric Association: Diagnostic and Statistical Manual of Mental Disorders, ed 4, Text Revision. Washington, American Psychiatric Press, 2002.

47 World Health Organization: International Classification of Diseases and Related Health Problems, ed 10. Genève, World Health Organization, 1992.

48 Onyike CU, Sheppard JM, Tschanz JT, Norton MC, Green RC, Steinberg M, WelshBohmer KA, Breitner JC, Lyketsos CG: Epidemiology of apathy in older adults: the Cache County Study. Am J Geriatr Psychiatry 2007; 15:365-375.

49 Brodaty H, Altendorf A, Withall A, Sachdev P: Do people become more apathetic as they grow older? A longitudinal study in healthy individuals. Int Psychogeriatr 2010;22:426436.
50 Ligthart SA, Richard E, Fransen NL, Eurelings LS, Beem L, Eikelenboom P, van Gool WA, Moll van Charante EP: Association of vascular factors with apathy in communitydwelling elderly individuals. Arch Gen Psychiatry 2012;69:636-642.

51 van der Mast RC, Vinkers DJ, Stek ML, Bek MC, Westendorp RG, Gussekloo J, de Craen AJ: Vascular disease and apathy in old age. The Leiden 85-Plus Study. Int J Geriatr Psychiatry 2008;23:266-271.

52 Ishii S, Weintraub N, Mervis JR: Apathy: a common psychiatric syndrome in the elderly. J Am Med Dir Assoc 2009;10:381-393.

- 53 Benoit M, Andrieu S, Lechowski L, GilletteGuyonnet S, Robert PH, Vellas B, REAL-FR group: Apathy and depression in Alzheimer's disease are associated with functional deficit and psychotropic prescription. Int J Geriatr Psychiatry 2008;23:409-414.

54 Ikeda M, Fukuhara R, Shigenobu K, Hokoishi K, Maki N, Nebu A, Komori K, Tanabe H: Dementia associated mental and behavioural disturbances in elderly people in the community: findings from the first Nakayama study. J Neurol Neurosurg Psychiatry 2004;75:146-148.

55 Treiber KA, Lyketsos CG, Corcoran C, Steinberg M, Norton M, Green RC, Rabins P, Stein DM, Welsh-Bohmer KA, Breitner JC, Tschanz JT: Vascular factors and risk for neuropsychiatric symptoms in Alzheimer's disease: the Cache County Study. Int Psychogeriatr 2008;20:538-553.

56 Jorge RE, Starkstein SE, Robinson RG: Apathy following stroke. Can J Psychiatry 2010; 55:350-354.

57 Robinson RG, Jorge RE, Clarence-Smith K, Starkstein S: Double-blind treatment of apathy in patients with poststroke depression using nefiracetam. J Neuropsychiatry Clin Neurosci 2009;21:144-151.

58 Nishio Y, Hashimoto M, Ishii K, Mori E: Neuroanatomy of a neurobehavioral disturbance in the left anterior thalamic infarction. J Neurol Neurosurg Psychiatry 2011;82: $1195-1200$

59 Ghika-Schmid F, Bogousslavsky J: The acute behavioral syndrome of anterior thalamic infarction: a prospective study of 12 cases. Ann Neurol 2000;48:220-227.

60 Caplan LR, Schmahmann JD, Kase CS, Feldmann E, Baquis G, Greenberg JP, Gorelick PB, Helgason C, Hier DB: Caudate infarcts. Arch Neurol 1990;47:133-143. 